\title{
A Study on RGB Image Multi-Thresholding using Kapur/Tsallis Entropy and Moth-Flame Algorithm
}

\author{
V. Rajinikanth ${ }^{1}$, Seifedine Kadry ${ }^{2}$, Rubén González Crespo ${ }^{3 *}$, Elena Verdú ${ }^{3}$ \\ ${ }^{2}$ Faculty of Applied Computing and Technology, Noroff University College, Kristiansand (Norway) \\ ${ }^{3}$ School of Engineering and Technology, Universidad Internacional de la Rioja (UNIR), Logroño \\ (Spain)
}

${ }^{1}$ Department of Electronics and Instrumentation Engineering, St. Joseph's College of Engineering, Chennai 600119, TN (India)

Received 16 May 2021 | Accepted 18 November 2021 | Published 24 November 2021

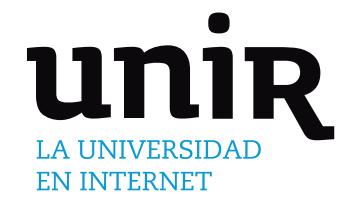

KEYWORDS

Finest Threshold, Kapur's Entropy, Tsallis Entropy, Moth-FlameOptimization, Picture Quality, PSNR, SSIM.

DOI: $10.9781 /$ ijimai.2021.11.008

\section{INTRODUCTION}

$\mathrm{B}$ I-LEVEL and multi-level image thresholding is widely employed to improve the quality of the image by grouping the pixels based on the selected threshold level. In literature, a number of image threshold methods are implemented on a class of gray/RGB scale images due to their practical significance. Recently, threshold methods are employed to pre-process a range of images with varied dimension and different pixel distributions [1], [2]. More commonly, the images, such as benchmark photographs [3]-[5], satellite images [6] and medical pictures [7] are thresholded with various methodologies and different optimization algorithms. A considerable number of thresholding methods, such as Otsu, Kapur, Tsallis, Renyi and Shannon are already implemented on a variety of digital images and every work discussed its own contribution. The review of the existing threshold identification can be found in [8], [9].

The concept used in the threshold operation is; adjusting the thresholds of the given image to enhance its features by grouping the similar pixels based on the chosen threshold value. This process is normally carried out with the help of computer algorithms and this procedure is terminated based on a chosen Objective Function (OF). In most of the cases, maximization of OF is preferred and the chosen

${ }^{*}$ Corresponding author

E-mail address: ruben.gonzalez@unir.net algorithm will continuously work to maximize the OF by adjusting the thresholds arbitrarily.

In most of the medical image processing techniques, the thresholding is chosen as a pre-processing procedure and the outcome in medical data assessment depends on the implemented threshold operation [10]. Further, entropy supported functions are widely used in medical data assessment [11]-[13] and hence, it is essential to identify the suitable entropy based technique to pre-process the gray/RGB scale picture [14]. Assessment of RGB scaled image is quite complex due to its complex histogram and the methodology which works well on RGB scale images can be easily transferred to pre-process the gray scale images. Further, the complexity of the image processing task also will increase based on the dimension of the image and hence, in this work the benchmark images with dimensions; $512 \times 512 \times 3$ and $720 \times 576 \times 3$ are considered for the demonstration.

The proposed work aims to evaluate the threshold performance of Kapur's/Tsallis entropy functions on a chosen RGB test picture. Both these entropy functions work by identifying and improving the essential pixels of the image which consist the key pixel groups. Threshold identification in RGB grade image is quite complex compared to the gray scale image, hence, this work employed the traditional MothFlame-Optimization (MFO) algorithm and the performance of the proposed technique is verified based on the computed Picture-QualityValues (PQV). The experimental outcome of this study confirms that the PQV achieved for Tsallis is better in RGB scaled images compared 
to Kapur. This result confirms that the RGB scale images thresholded with Tsallis helps to achieve a better pixel grouping and this procedure can be considered to examine the traditional and medical grade digital pictures to get better results during the examination.

The proposed work considered the following procedures to improve the outcome of thresholding:

- Implementing the bounded search procedure discussed by Raja et al. [15] to minimize the search time.

- Implementing the modified objective function discussed in Rajinikanth and Couceiro [16] to enhance the outcome.

- Experimental investigation is executed on a commonly used RGB scale benchmark images with two different dimensions.

\section{RElATEd EARLIER WORKS}

Image multi-thresholding is one of the common techniques to enhance the test image with a chosen procedure. The entropy based methods are normally considered in the literature to enhance the vital information in the chosen test picture. When a medical image (Gray/ RGB) is to be processed, the entropy based thresholding helps to provide better pixel grouping, which improves the visibility of the abnormal region of the image, which is to be examined. Implementation of entropy supported medical image enhancement is common procedure and Kapur's/Tsallis thresholding schemes are widely employed in this task. The Kapur's/Tsallis based medical image thresholding can be found in the earlier research work [10].

Examination of gray scale picture is quite simple and a manual threshold selection procedure can also be employed to improve the quality of the gray scale picture. The assessment of the RGB scaled image is one of the complex tasks due to its complex histogram and hence, a considerable number of procedures are developed to evaluate the RGB scale pictures.

Table I summarizes few recently implemented entropy based thresholding techniques employed to threshold the RGB images. The considered RGB image thresholding procedure helps to improve the information in the chosen test picture by grouping the similar pictures and most of the recently developed RGB thresholding procedures employed a chosen heuristic algorithm to identify the threshold value automatically by maximizing the entropy value.
The information presented in Table I confirms that the multi-level thresholding with entropy functions are a widely adopted procedure, which provides a significant result on a class of traditional and medical grade images. The entropy supported medical image examination is a widely adopted pre-processing technique in which the thresholding helps to enhance the abnormal/disease section in the image, which is then extracted and assessed with a chosen segmentation method. The multi-level thresholding with $\mathrm{Th}=2,3,4,5$ is a commonly adopted method and the results of earlier works presented in Table I confirms that the entropy supported thresholding helps to get a better PQV compared to Otsu's between class variance technique. Hence, this work employed the entropy supported thresholding to enhance the RGB scaled pictures with varied dimensions.

\section{Methodology}

The heuristic algorithm based threshold identification has largely attracted the research community to pre-process the gray/RGB scale images, due to its wide use. The methodology, which works well on a class of RGB grade picture, will work on a class of gray scale pictures. The threshold methodology implemented in this work is depicted in Fig. 1 and this work considered the RGB pictures of dimension $512 \times 512 \times 3$ and $720 \times 576 \times 3$ pixels.

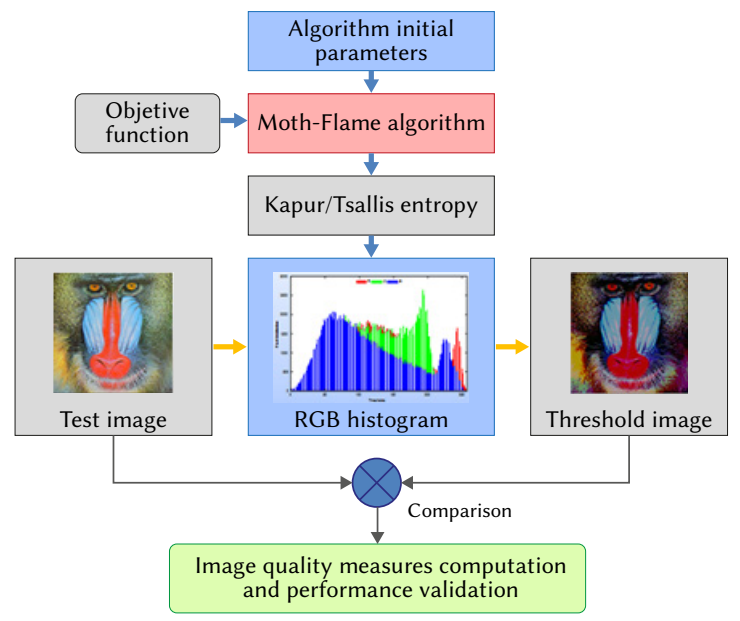

Fig. 1. Outline of methodology executed in the proposed work.

TABle I. Summary of the RGB Scaled Image Thresholding With Entropy Function and Heuristic Algorithm

\begin{tabular}{|l|l|}
\hline Reference & Methodology \\
\hline Aziz et al. [1] & $\begin{array}{l}\text { Multi-thresholding using Wale algorithm and MFO is implemented to find the optimal thresholds for a class of benchmark } \\
\text { gray scale images. }\end{array}$ \\
\hline Jia et al. [2] & Implementation of RGB image thresholding is presented using modified MFO. \\
\hline Sathya et al. [17] & $\begin{array}{l}\text { Implementation of RGB image thresholding using Kapur, Otsu and minimum cross entropy is presented using heuristic } \\
\text { technique. }\end{array}$ \\
\hline Farshi and Ardabili [18] & Implementation of multi-level thresholding using hybrid algorithm is presented using benchmark pictures. \\
\hline Anitha et al. [19] & RGB image thresholding with modified whale optimization is presented for benchmark test pictures. \\
\hline Kurban et al. [20] & A detailed review on RGB image thresholding for aerial images. \\
\hline Bhandari [21] & Beta differential evolution algorithm supported fast RGB image thresholding is presented using benchmark pictures. \\
\hline Xing [22] & RGB image multi-thresholding with improved emperor penguin optimization is presented. \\
\hline Meyyappan et al. [23] & Skin melanoma image thresholding using Kapur's entropy and harmony search algorithm is demonstrated. \\
\hline Borjigin and Sahoo [24] & RGNB image thresholding with Tsallis entropy is discussed. \\
\hline Kadry and Rajinikanth [25] & Implementation of Tsallis entropy and MFO is presented for gray scale image thresholding. \\
\hline Elaziz et al. [26] & Multilevel thresholding of benchmark pictures are presented using whale optimization algorithm. \\
\hline Elaziz et al. [27] & Multi-level thresholding with Harris hawks algorithm is demonstrated. \\
\hline
\end{tabular}


Initially, a chosen threshold method with a chosen optimization algorithm is implemented to enhance the considered test picture. The implemented method will randomly adjust the threshold of the picture, till the objective function value is maximized or a maximum number of iterations is reached. After getting the pre-processed image, the performance of this image is then computed based on a comparative analysis with the original picture. During this process, the essential PQVs are computed and, based on these values; the performance of the implemented threshold operation is confirmed.

Executing the multi-thresholding on a chosen test picture requires the following procedures:

- Choice of appropriate thresholding methodology.

- Choice of the heuristic technique.

- Selection of the objective function.

- Selection of PQV to assess the outcome of the experiment.

\section{A. Entropy Function}

Entropy based methods are largely used in image as well as signal evaluation methods to identify the abnormal regions. The earlier research works confirmed that the entropy based procedures offers better results compared to other similar methodologies.

In image examination applications, Kapur's Entropy (KE) and Tsallis Entropy (TE) are widely employed in situations when the key pixel groups in the image are to be enhanced based on a chosen threshold [10]. The processing methodology related to the KE is simple and it can be mathematically denoted using a simple probability distribution function. The execution steps in TE are quite complex and its probability function is monitored using an entropic index. This research work aims to evaluate the thresholding performance of $\mathrm{KE}$ and TE on chosen benchmark RGB scale images.

\section{Kapur's Entropy}

KE was initially proposed by Kapur et al. [28] to pre-process a class of gray scale images. In this work, the histogram values are randomly adjusted till the entropy of the histogram reaches a maximized value.

The KE for a gray scale image is mathematically described as follows:

Let, $T h=\left[T_{1}, T_{2}, \ldots, T_{n-1}\right]$ denote the threshold vector for gray picture andfor the case of the RGB, itcanberepresentedas; $T h_{R}=\left[T_{R 1}, T_{R 2}, \ldots, T_{R n-1}\right]$, $T h_{G}=\left[T_{G 1}, T_{G 2}, \ldots, T_{G n-1}\right]$ and $T h_{B}=\left[T_{B 1}, T_{B 2}, \ldots, T_{B n-1}\right]$.

The alteration in threshold is done, till the following condition is maximized:

$$
J_{K E_{\text {max }}=F(T h)}=\sum_{j=1}^{n} H_{j}^{C}
$$

where $H_{j}^{C}=$ probability distribution and $\mathrm{C}=$ image class identification ( $C=1$ for gray image and $\mathrm{C}=3$ for $\mathrm{RGB}$ ).

The KE described in this section can be adopted for the RGB scale images, in which the $R, G$ and $B$ histogram is separately determined with the chosen function and this evaluation is continued till the average entropy value for the image is maximized $\left(J_{K E_{\max }}\right)$. Other related information for the KE can be found in [2], [10].

\section{Tsallis Entropy}

The idea of TE was derived from Shannon's Function (SF) depicted in Eqn. (2) and this equation forms the SF when $\varepsilon \rightarrow 1$.

$$
S F=\frac{1-\sum_{j=1}^{T h}\left(p_{j}\right)^{\varepsilon}}{\varepsilon-1}
$$

where $T h=$ total thresholds and $\varepsilon=$ entropy indicator.

The simulated additively rule for the entropy is as follows:
For a gray scale picture with a threshold of range $[0,1, \ldots, L-1]$ along with probability distributions $P_{i}=P_{0}, P_{1}, \ldots, P_{L-1}$, it is considered to identify the final entropy function based on assigned threshold.

For the RGB class image, this distribution is as follows; $P_{R i}=P_{R 0}, P_{R 1}$, $\ldots, P_{R L-1}, P_{G i}=P_{G 0^{\prime}} P_{G 1}, \ldots, P_{G L-1}$ and $P_{B i}=P_{B 0^{\prime}} P_{B 1}, \ldots, P_{B L-1}$.

Compared to the $\mathrm{KE}$, the execution of the TE is quite complex, since, its outcome depends mainly on the probability distributions, which decide the maximized entropy value. Other related details of TE can be found in the earlier works [25], [29].

\section{B. Moth-Flame-Optimization}

In the literature, a number of Heuristic Algorithms (HA) are proposed and implemented by the researchers to find the optimal solution for a class of real world problems. The performance of a chosen HA depends on its updating mechanism and in most of the existing algorithms, the movement of agents from the current location to the new location happens randomly. For a chosen problem, the search of the optimal solution depends on the dimension of the solution and the search methodology which moves the agents towards the optimal solution. Most of the existing HA considered random search processes to move the agents towards the optimal location. Further, the search procedures, such as chaotic search, Lévy-flight, and Brownian-distribution are also adopted to move the agents towards the finest solution with lesser iteration. Recently, to move the search agents quickly towards the optimal solution, the spiral and spherical search strategies are proposed to get the optimal solution to a chosen problem [1], [2], [25].

MFO is a nature inspired HA invented by Mirjalili in 2015 [30] to find the optimal solution for numerical benchmark problems. The concept of MFO is based on the movement of a Moth towards Flame based on a pre-defined pattern (spiral). In this algorithm, the moths are the search agents and the flame is the solution for the problem. If the algorithm search is initiated with a number of agents (moths), then every agent is allowed to reach their associated flame (solution) using a predefined defined search-pattern. The conventional MFO with a single agent searching the solution in a 3D space is depicted in Fig. 2. All the agents are randomly initiated in the search universe based on the dimension of the search and every agent is allowed to converge towards the solution when the search iteration increases. The main merit of this algorithm is that every agent travels in a spiral shaped search path, which helps to reduce the number of iterations to find the optimal solution compared to the random, Lévy-flight, and Brownian-distribution functions. Further, the search pattern of MFO is predefined and it does not take the arbitrary path to reach the solution during an optimization task and this procedure makes the MFO more successful in finding the solutions for a chosen problem compared to the existing algorithms in the literature.

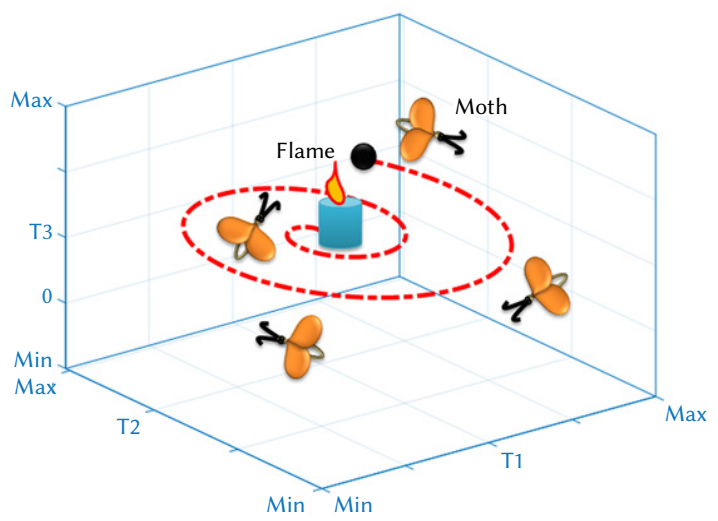

Fig. 2. Search pattern of a Moth towards a Flame. 
TABle II. Heuristic Algorithms and their Parameters Considered in This Work to Justify the Performance of MFO

\begin{tabular}{|c|c|c|c|c|c|c|}
\hline Measures & PSO [6] & BA [4] & FA [16] & MA [37] & AOA [38] & MFO \\
\hline Number of agents & 20 & 20 & 20 & 20 & 20 & 20 \\
\hline Search dimension & $\mathrm{Th}=2,3,4,5$ & $\mathrm{Th}=2,3,4,5$ & $\mathrm{Th}=2,3,4,5$ & $\mathrm{Th}=2,3,4,5$ & $\mathrm{Th}=2,3,4,5$ & $\mathrm{Th}=2,3,4,5$ \\
\hline Search pattern & Random search & Ikeda-Map & Lévy-flight & Lévy-flight & $\begin{array}{l}\text { Multiple search } \\
\text { pattern }\end{array}$ & $\begin{array}{l}\text { Spiral combined } \\
\text { with random } \\
\text { search }\end{array}$ \\
\hline Search termination & Iter $_{\max }$ & Iter $_{\max }$ & Iter $_{\max }$ & Iter $_{\max }$ & Iter $_{\max }$ & Iter $_{\max }$ \\
\hline
\end{tabular}

To define the mathematical model for MFO; let us consider that a chosen search space has a-number of moths $(\mathrm{M})$ and b-number of flames (F). Let the initial distance among $\mathrm{M}$ and $\mathrm{F}$ is $D_{a}=\left|F_{b}-M_{a}\right|$, which is to be minimized to find the optimal solution.

The mathematical expression for a moth progress towards the flame can be defined as:

$$
M_{a}=D_{a} * e^{\kappa \Re} * \cos (2 \pi \Re)+F_{b}
$$

where $M_{a}=\mathrm{a}^{\text {th }}$ moth, $F_{b}=\mathrm{b}^{\text {th }}$ flame, $\kappa=$ constant to define spiral pattern, and $\Re=$ random variable of range $[-1,1]$.

In this equation, the random variable $\Re$ can be used to speed up the spiral shape formation process to guide the Moths towards the flame. The complete information about the traditional and improved MFO can be found in [1], [2], [25], [31]-[33].

\section{Implementation}

In this work, the MFO algorithm is considered to identify the optimal threshold for the RGB scaled images using the KE/TE based on a chosen objective function. Initially, the test image is pre-processed based on a chosen threshold $(\mathrm{Th}=2,3,4,5)$ by maximizing the entropy value. After thresholding the image based on a chosen Th, a pixel wise comparison is executed to confirm the enhancement in the thresholded image.

In the literature, multi-level thresholding with Single-ObjectiveFunction (SOF) and weighted sum of Multiple-Objective-Function (MOF) is very common and the earlier work confirmed that the MOF helps to get better enhancement compared to the SOF. Hence, this work employed the MOF to threshold the RGB scale image with $\mathrm{KE}$ / TE.

The MOF considered in this work is depicted in Eqn. (4):

$$
M O F_{\text {max }}=\left(W_{1} * \text { Entropy }\right)+\left(W_{2} * P S N R\right)+\left(W_{3} * S S I M\right)
$$

where, Entropy = maximized KE/TE, PSNR = Peak signal-to-noise ratio, SSIM = Structural Similarity Index $W_{1}=1, W_{2}=0.5$, and $W_{3}=0.5$.

In this work, HA search with KE/TE is performed to satisfy Eqn. (4).

\section{Picture-Quality-Value Computation}

The performance of the image processing scheme is confirmed by computing the image related measures. From the image thresholding literature, it is noted that the merit of the image thresholding process can be confirmed by computing the necessary PQV. This PQV can be obtained by comparing the original and threshold image.

The quality of the thresholding can be confirmed by computing the measures, such as Mean Squared Error (MSE), Root MSE (RMSE), PSNR, Average Difference (AD), Structural Content (SC), Normalized Absolute Error (NAE) and SSIM.
All these measures help to confirm that the thresholded image is having better enhancement compared to the original test picture. MSE and RMSE are the measures of the intensity variation in the processed image compared to the original picture. The PSNR provides the ratio among the highest potential pixel of an image and the power of distorting noise which affects the quality of threshold image. The $\mathrm{AD}$ presents the possible deviation among the original and processed image. SC confirms the presence of the vital information in the threshold image compared to the original image. NAE is the difference between the original and processed picture and SSIM presents the image quality degradation due to thresholding.

Let us consider that $\mathrm{O}$ and $\mathrm{P}$ denote the dimension of real $(\mathrm{R})$ and threshold $(\mathrm{T})$ pictures, and the mathematical expression of these measures can be expressed as:

$$
\begin{aligned}
& \mathrm{MSE}=\frac{1}{O P} \sum_{j=1}^{O} \sum_{n=1}^{P}\left(R_{j, n}-T_{j, n}\right) \\
& \mathrm{RMSE}=\sqrt{M S E} \\
& \mathrm{PSNR}=10 \log \frac{(255)^{2}}{M S E} \\
& \mathrm{AD}=\frac{\sum_{j=1}^{O} \sum_{n=1}^{P}\left(R_{j, n}-T_{j, n}\right)}{O P} \\
& \mathrm{SC}=\frac{\sum_{j=1}^{O} \sum_{n=1}^{P} R_{j, n}}{\sum_{j=1}^{O} \sum_{n=1}^{P} T_{j, n}} \\
& \mathrm{NSE}=\sum_{j=1}^{O} \sum_{n=1}^{P}\left(R_{j, n}-T_{j, n}\right) \\
& \mathrm{SSIM}=\frac{\left(2 \mu_{o} \mu_{p}+G_{1}\right)\left(2 \sigma_{o p}+G_{2}\right)}{\left(\mu_{o}^{2}+\mu_{p}{ }^{2}+G_{1}\right)\left(\sigma_{o}{ }^{2}+\sigma_{p}{ }^{2}+G_{2}\right)}
\end{aligned}
$$

where, $\mu_{o}$ and $\mu_{p}$ are mean values, $\sigma_{o}^{2}$ and $\sigma_{p}^{2}$ variances and $\sigma_{o p}$ correlation coefficient. Further, $G_{1}=(0.01 \mathrm{~L})^{2}$ and $G_{2}=(0.03 \mathrm{~L})^{2}$.

Other information on these PQVs can be found in the literature [34]-[36].

\section{E. Performance Evaluation}

The performance of the proposed thresholding by MOA based KE/ TE is then validated using the well known HA, such as Particle Swarm Optimization (PSO), Bat Algorithm (BA), Firefly Algorithm (FA), Mayfly Algorithm (MA) and Aquila Optimization algorithm (AOA). To have a fair evaluation, every algorithm is assigned with similar agents, search dimension, objective function, Iter $_{\max }$ and termination as depicted in Table II. Every algorithm has its own search pattern which influences the search convergence and the attained image quality. Compared to $\mathrm{MA}$ and $\mathrm{AOA}$, the implementation steps involved in MFO are quite simple, Further, the PSO, BA and FA follow a complex search pattern and hence the results by the MFO are satisfactory on the chosen test images. 


\section{Results AND Discussions}

This work aims to demonstrate a multi-threshold scheme for a class of images with varied dimensions. The experimental investigation is performed using MATLAB software and the proposed technique is independently tested on all the considered imagery with a threshold value ranging from 2 to 5 . This work executed an entropy based methodology to find the finest threshold with the help of MFO algorithm.

Later an assessment of the threshold image and original picture is performed to find the PQVs and based on these values; the performance of this system is validated. Primarily, this work is tested on the benchmark RGB pictures shown in Fig. 3. This figure presents the chosen trial images (Fig. 3(a)) along with its histogram (Fig. 3(b)). The histogram of RGB images is very complex and hence, identification of the finest thresholds is quite difficult. Hence, this work implemented a bounded threshold technique discussed by Raja et al. [15]. In bounded search, instead of keeping $T h_{\text {min }}=0$ and $T h_{\max }=255=\mathrm{L}-1$, a boundary is assigned based on its pixel strength.
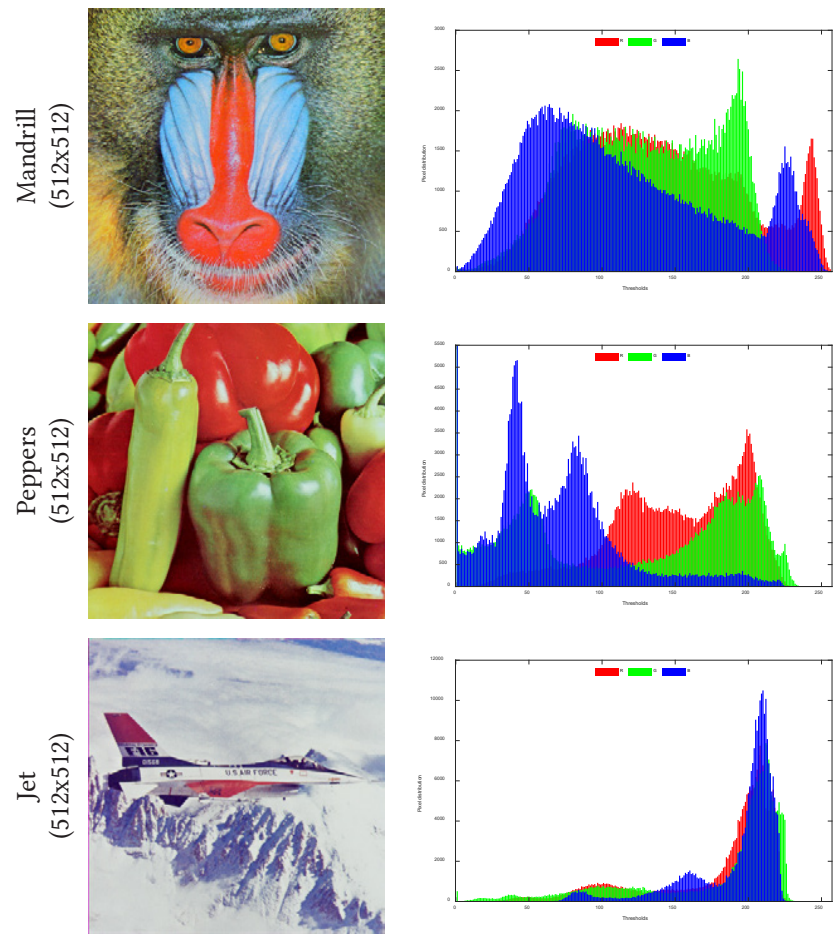

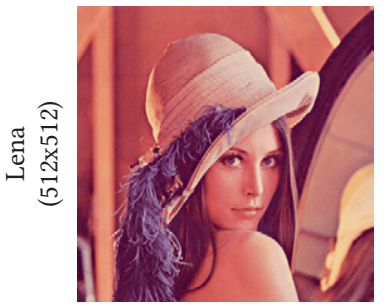

(a)

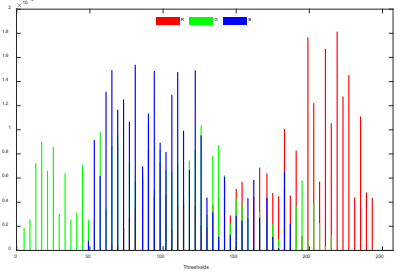

(b)
Fig. 3. Chosen digital photographs of dimension 512x512x3.

The bounded search is executed separately on R, G and B thresholds as follows:

$$
\begin{aligned}
R_{\text {channel }} & =T h_{\text {min }}<R<T h_{\text {max }} \\
G_{\text {channel }} & =T h_{\text {min }}<G<T h_{\text {max }} \\
B_{\text {channel }} & =T h_{\text {min }}<B<T h_{\text {max }}
\end{aligned}
$$

If the minimum and maximum thresholds are assigned for each channel, then it is easy for the MFO to find the finest threshold with minimal iteration.

Fig. 4 depicts the R,G,B histogram for Mandrill image, for which the threshold search boundary is assigned as follows:

$$
\begin{gathered}
R_{\text {channel }}=28<R<248 \\
G_{\text {channel }}=20<G<216 \\
B_{\text {channel }}=8<B<245
\end{gathered}
$$

The bounded search helped to achieve satisfactory results with lesser search iteration. In this work a ten-fold cross validation is implemented for every image with every threshold and the best result among them is considered as the optimized threshold.
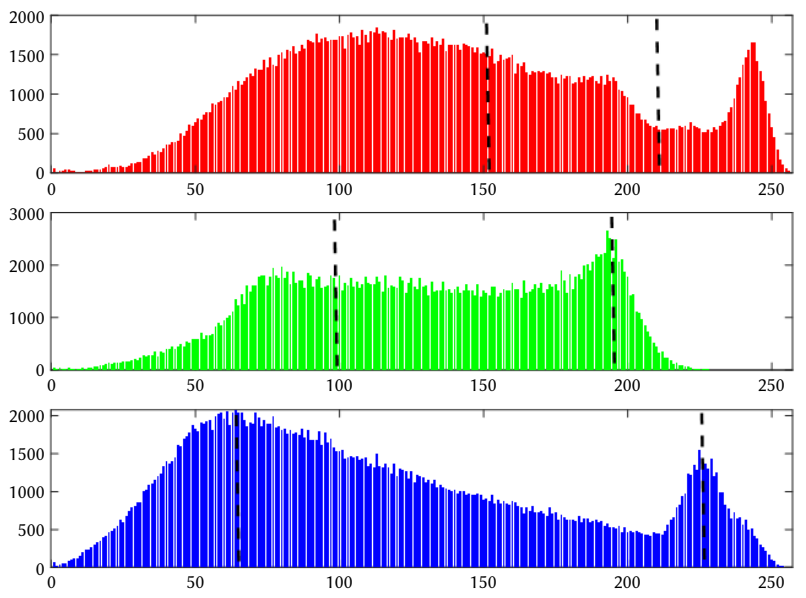

Fig. 4. Bi-level threshold implemented on RGB scale histogram.

Initially, the KE based multilevel thresholding is implemented using Mandrill picture for a chosen Th of 2 to 5. For this image, every algorithm helped to identify similar objective value with approximately similar PQV. But the MFO showed a steady search behavior due to its spiral search operator compared to other algorithms. The search convergence by the FA and MA is better compared to the MFO due to its Lévy-flight process and compared to PSO, BA and AOA, the search convergence of MFO is better. The performance measures computed for the Mandrill with $\mathrm{KE}$ and $\mathrm{Th}=5$ is presented in Fig. 5, which confirms that the overall $\mathrm{PQV}$ obtained by the MFO is satisfactory compared to other HA. Fig. 6 presents a performance comparison of MFO with other methods and this comparison confirms that the MFO's performance is better compared to PSO, BA and FA and approximately similar to MA and AOA.

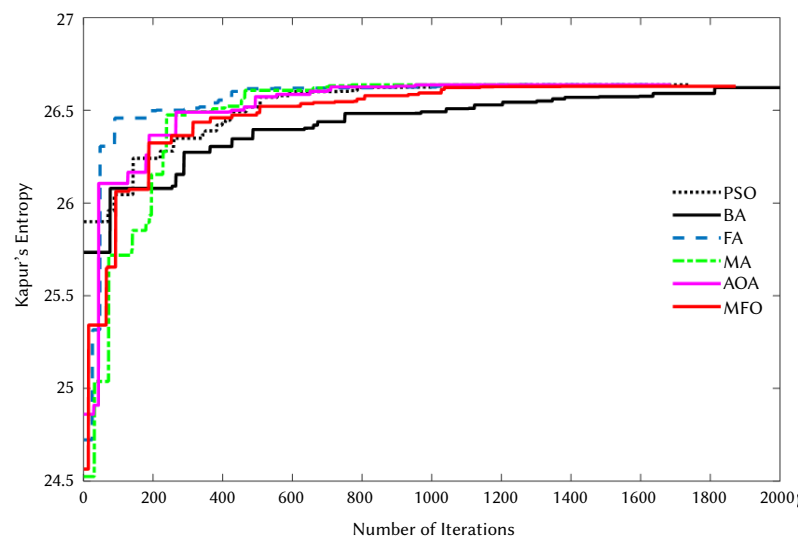

Fig. 5. Search convergence of chosen HA for Mandrill with KE and Th=5. 


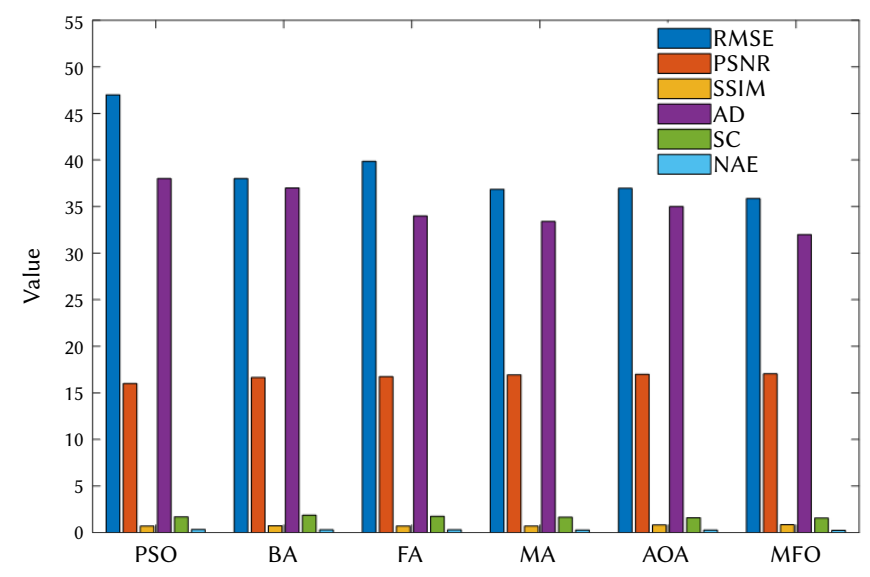

Fig. 6. Performance evaluation of MFO with other HA.

The results attained with the MFO and the KE $(\mathrm{MFO}+\mathrm{KE})$ are depicted in Fig. 7 for the chosen thresholds. Fig. 7(a) to (d) depict the results attained for $\mathrm{Th}=2$ to 5 , respectively. After recording the thresholded image, an assessment of this image with its original image is performed to compute all the possible PQVs discussed in sub-section III.D.

A pixel wise comparison among the images is performed to compute the essential PQVs for each image. Initially, this computation is implemented for the Mandrill image and the attained SSIM map is depicted in Fig. 8. Other PQVs attained with this procedure are presented in Table III. The values of this table confirm that, the lesser threshold gives lower values of the PQVs and this value gradually improves for higher values of threshold.

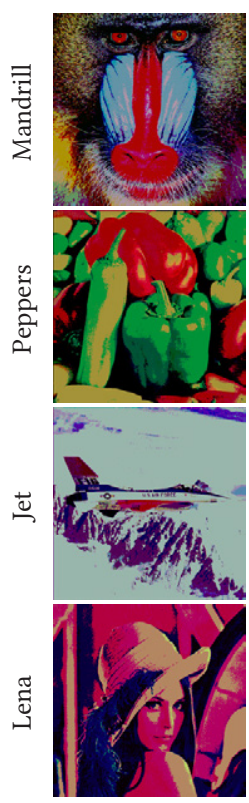

(a)

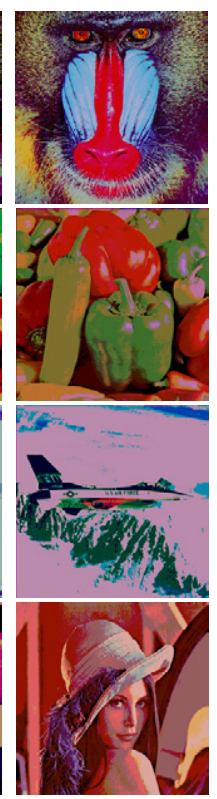

(b)

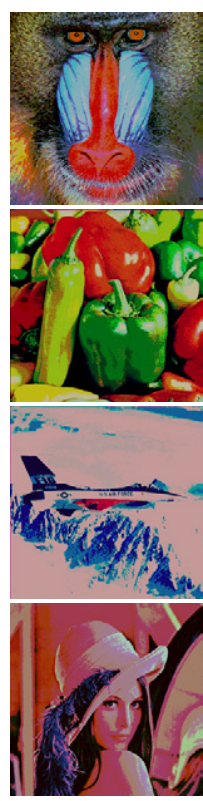

(c)

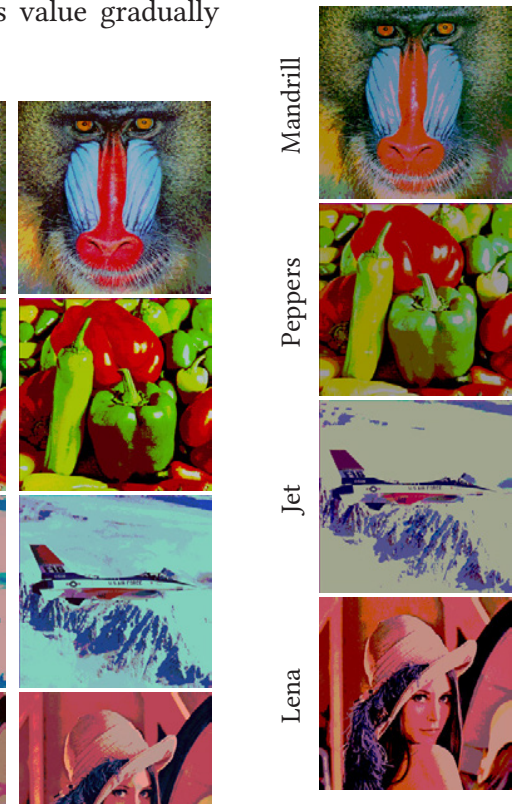

(a)

TABLE III. PQVs AtTAined With MFO+KE

\begin{tabular}{|c|c|c|c|c|c|c|c|}
\hline Image & Th & RMSE & PSNR & SSIM & $\mathrm{AD}$ & SC & NAE \\
\hline \multirow{4}{*}{ 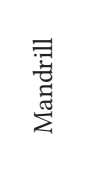 } & 2 & 63.9934 & 12.0081 & 0.6168 & 58.7727 & 2.2679 & 0.4534 \\
\hline & 3 & 46.4955 & 14.7826 & 0.7614 & 40.1494 & 1.6591 & 0.3098 \\
\hline & 4 & 41.4216 & 15.7863 & 0.7897 & 37.7085 & 1.7388 & 0.2909 \\
\hline & 5 & 35.8594 & 17.0387 & 0.8427 & 31.9953 & 1.5582 & 0.2469 \\
\hline \multirow{4}{*}{ 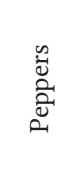 } & 2 & 51.4619 & 13.9011 & 0.6193 & 48.2072 & 2.2920 & 0.4010 \\
\hline & 3 & 44.5729 & 15.1494 & 0.6700 & 38.7395 & 2.1937 & 0.3222 \\
\hline & 4 & 43.8707 & 15.2873 & 0.6878 & 37.3242 & 1.6947 & 0.3105 \\
\hline & 5 & 40.5123 & 15.9791 & 0.7889 & 34.9152 & 1.6760 & 0.2904 \\
\hline \multirow{4}{*}{$\stackrel{\Xi}{\varrho}$} & 2 & 54.5974 & 13.3874 & 0.8085 & 52.8337 & 1.8570 & 0.2949 \\
\hline & 3 & 45.6481 & 14.9424 & 0.8247 & 43.7349 & 1.6415 & 0.2441 \\
\hline & 4 & 44.3914 & 15.1848 & 0.8555 & 40.2827 & 1.5176 & 0.2248 \\
\hline & 5 & 33.4113 & 17.6529 & 0.9075 & 31.9359 & 1.4242 & 0.1782 \\
\hline \multirow{4}{*}{ 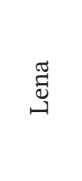 } & 2 & 64.6375 & 11.9211 & 0.4769 & 60.4672 & 2.6461 & 0.5021 \\
\hline & 3 & 36.7225 & 16.8322 & 0.7460 & 34.1909 & 1.7332 & 0.2839 \\
\hline & 4 & 35.8501 & 17.0410 & 0.7827 & 32.9572 & 1.6923 & 0.2737 \\
\hline & 5 & 33.7777 & 17.5582 & 0.7891 & 31.0726 & 1.6477 & 0.2580 \\
\hline
\end{tabular}

A similar procedure is then repeated using the TE (MFO+TE) on the considered test images and the corresponding results are depicted in Fig. 9 and Table IV. Fig. 9(a) to (d) presents the results when the chosen thresholds are from 2 to 5 .

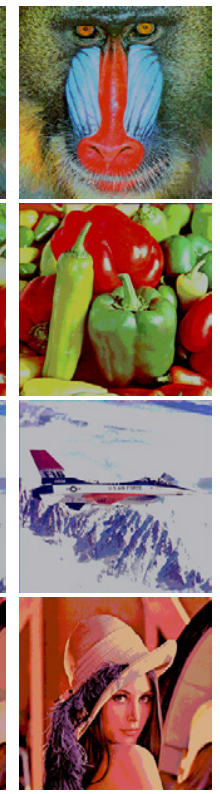

(c)

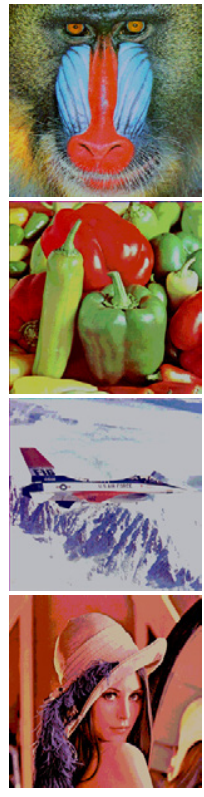

(d) (d)

Fig. 7. Thresholding outcome with $\mathrm{MFO}+\mathrm{KE}$

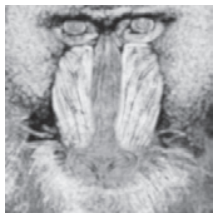

(a)

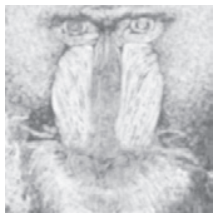

(b)

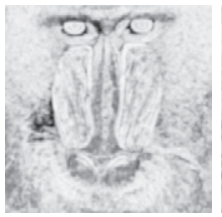

(c)

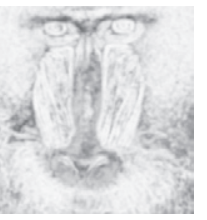

(d)
Fig. 8. SSIM map computed for Mandrill image.
Finally, an analysis between Table III and Table IV is then executed to assess the PQVs attained with MFO+KE and MFO+TE and this comparison confirms that, the results attained with TE are superior for all the thresholds compared to the KE. Even though the PQVs of $\mathrm{KE}$ are lesser, the pixel grouping and the image enhancement achieved with the KE is superior compared to the TE.

Hence, during the real image examination tasks, the choice of a particular technique can be done based on PQVs or based on image enhancement. During the real image processing task, the choice and implementation of a particular thresholding procedure depends on the operator and its expertise. 


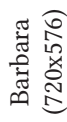
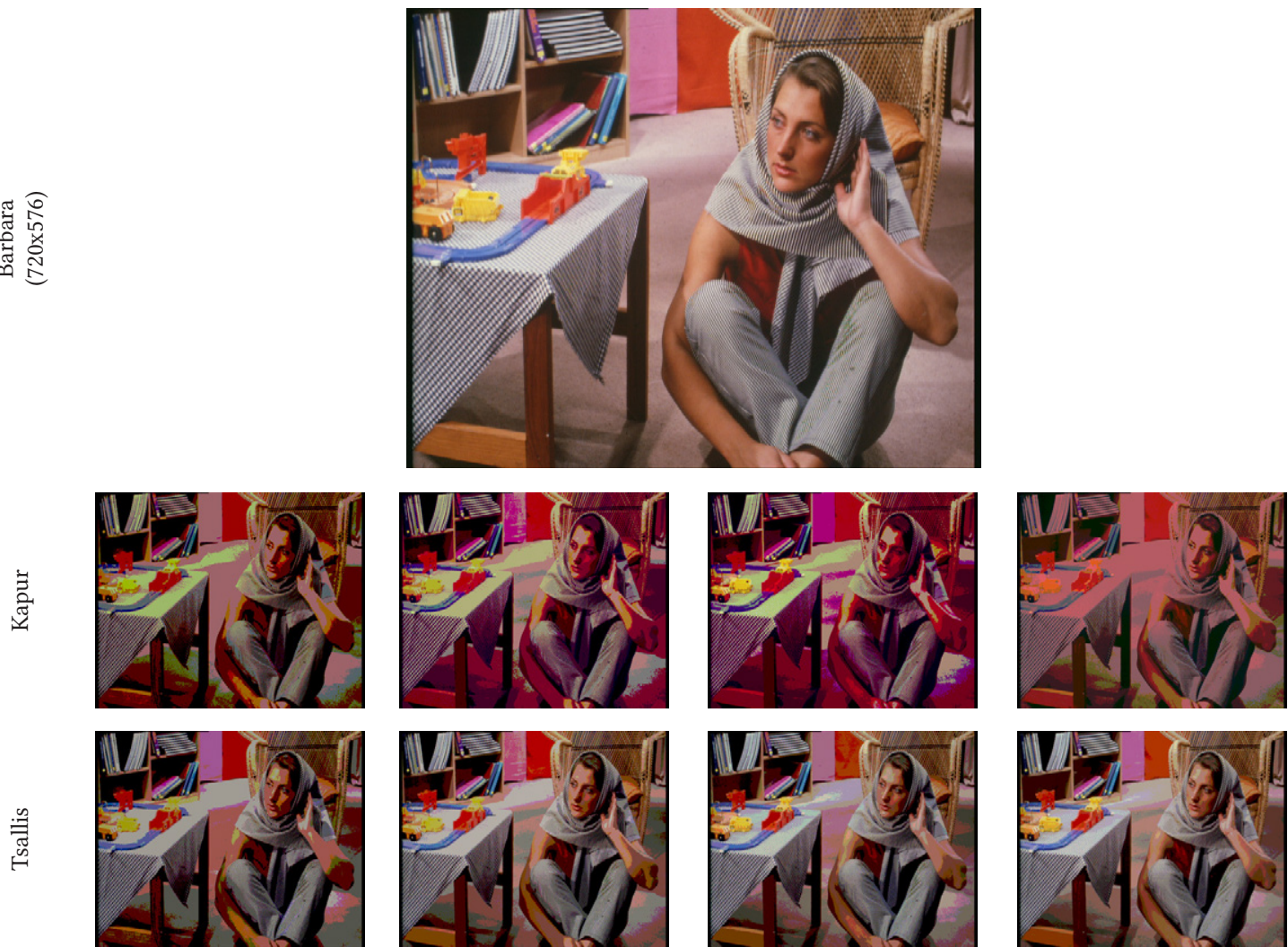

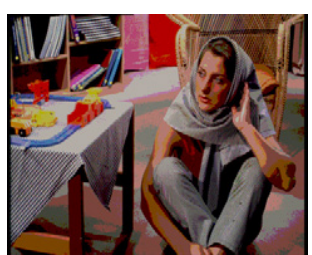

(b)

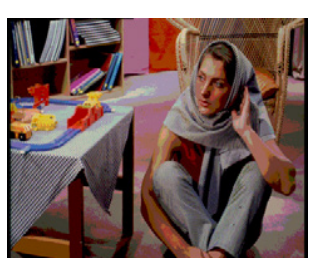

(c)

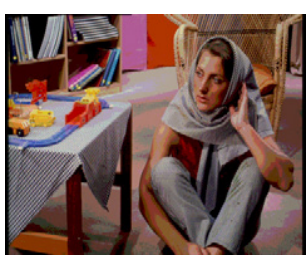

(d)

Fig. 10. Thresholding results for Barbara image.
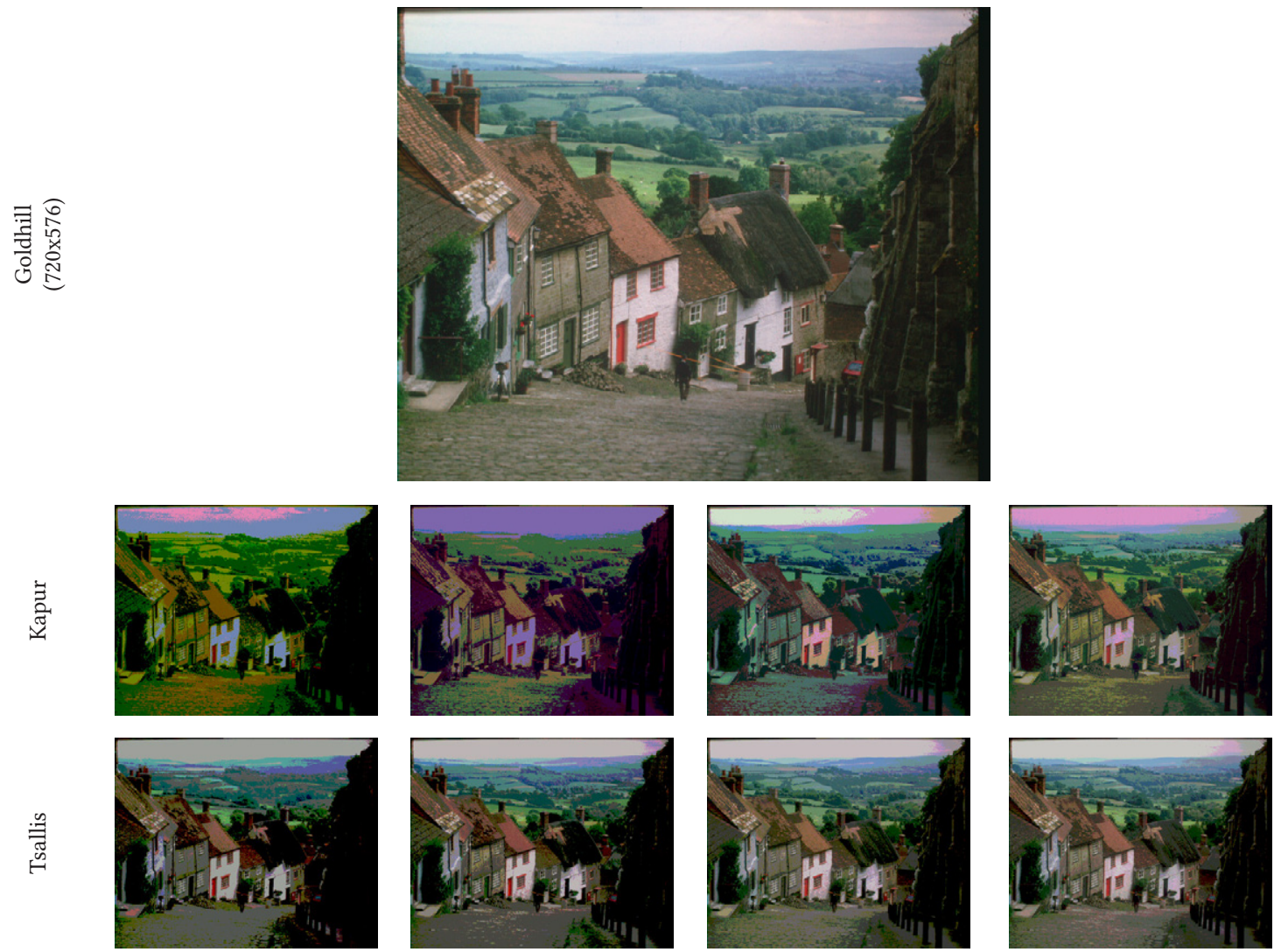

(b)

(c)

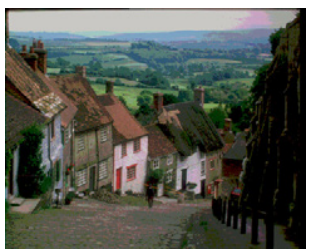

(a)

Fig. 11. Thresholding results for Goldhill picture. 
TABLE IV. PQVs Computed For The OUtCome of MFO+TE

\begin{tabular}{|c|c|c|c|c|c|c|c|}
\hline Image & Th & RMSE & PSNR & SSIM & $\mathrm{AD}$ & SC & NAE \\
\hline \multirow{4}{*}{ 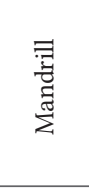 } & 2 & 42.9311 & 15.4754 & 0.8161 & 40.8138 & 1.9045 & 0.3149 \\
\hline & 3 & 30.7935 & 18.3616 & 0.8955 & 28.9921 & 1.5468 & 0.2237 \\
\hline & 4 & 24.8261 & 20.2326 & 0.9308 & 23.2634 & 1.4127 & 0.1795 \\
\hline & 5 & 20.7107 & 21.8069 & 0.9496 & 19.3494 & 1.3278 & 0.1493 \\
\hline \multirow{4}{*}{ 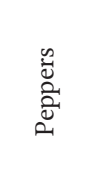 } & 2 & 40.0633 & 16.0759 & 0.7543 & 37.6425 & 1.8912 & 0.3131 \\
\hline & 3 & 30.6295 & 18.4080 & 0.8263 & 28.0955 & 1.5433 & 0.2337 \\
\hline & 4 & 25.1771 & 20.1107 & 0.8568 & 22.8180 & 1.3914 & 0.1898 \\
\hline & 5 & 18.8785 & 22.6115 & 0.9069 & 17.2850 & 1.2982 & 0.1438 \\
\hline \multirow{4}{*}{$\stackrel{ \pm}{\varrho}$} & 2 & 43.8722 & 15.2870 & 0.6715 & 41.6883 & 1.6468 & 0.2327 \\
\hline & 3 & 29.2309 & 18.8140 & 0.7817 & 27.2913 & 1.3549 & 0.1523 \\
\hline & 4 & 24.4745 & 20.3565 & 0.8020 & 22.8037 & 1.2900 & 0.1273 \\
\hline & 5 & 21.2613 & 21.5790 & 0.8194 & 19.6579 & 1.2465 & 0.1097 \\
\hline \multirow{4}{*}{ 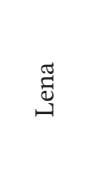 } & 2 & 41.1201 & 15.8497 & 0.6715 & 37.9305 & 1.7185 & 0.3150 \\
\hline & 3 & 31.1058 & 18.2740 & 0.7817 & 28.8739 & 1.5080 & 0.2398 \\
\hline & 4 & 27.1371 & 19.4595 & 0.8020 & 24.4986 & 1.3750 & 0.2034 \\
\hline & 5 & 26.5207 & 19.6591 & 0.8194 & 23.9857 & 1.3641 & 0.1992 \\
\hline
\end{tabular}

This work demonstrated a satisfactory result with the KE and better result with the TE with respect to the PQVs on image with dimension $512 \times 512 \times 3$ pixels and in future, the proposed approach can be used to test other existing benchmark gray/RGB class pictures.

A similar procedure is then executed for the test images with dimension $720 \times 576 \times 3$ pixels and the corresponding outcomes are depicted in Fig. 10 and Fig. 11 for various thresholds with chosen entropy values.

The results of the proposed study confirm that, the proposed thresholding work helps to attain better results on a class of RGB images. Further, the proposed work confirms that the overall performance of the TE based thresholding on RGB image is better compared to KE.

The future scope of this work is as follows:

- The proposed work can be employed to threshold the gray scale images.

- The MOF value can be enhanced by including more PQVs.

- This work currently implemented a traditional MFO algorithm which works based on a random operator, $\Re$ whose value varies with a range $[-1,1]$. In future, this random variable can be replaced with various search operators (Ex. Levy, Brownian-distribution and chaotic operator) to enhance the convergence of MFO.

\section{Conclusion}

This research aims to recommend a methodology to solve the multi-thresholding problem of RGB scale images using entropy value. This scheme used the thresholds ranging from 2 to 5 and to realize the optimum threshold, MFO is employed. This work proposes a random search along with a novel Multiple-Objective-Function (MOF). The role of MFO is to randomly adjust the thresholds till the MOF is maximized. This work is separately tested with KE and TE techniques on the chosen benchmark images. After discovering the necessary threshold for the chosen picture, in order to validate its performance, a comparison is performed among the original and threshold image to find the PQVs. Based on these values, the performance is confirmed. From the attained results, it is established that the proposed system works well on a class of RGB images with different dimensions. The experimental outcome confirms that the PQVs achieved with the TE are better compared to the KE. Further, a comparative assessment with KE confirmed that the performance of MFO is better compared to PSO, BA and FA and approximately similar to MA and AOA.

\section{REFERENCES}

[1] M.A.E. Aziz, A.A. Ewees, A.E. Hassanien, "Whale Optimization Algorithm and Moth-Flame Optimization for multilevel thresholding image segmentation," Expert Systems with Applications, vol. 83, pp. 242256, 2017, https://doi.org/10.1016/j.eswa.2017.04.023.

[2] H. Jia, J. Ma, W. Song, "Multilevel Thresholding Segmentation for Color Image Using Modified Moth-Flame Optimization," IEEE Access, vol. 7, pp. 44097- 44134, 2019, DOI: 10.1109/ACCESS.2019.2908718.

[3] V. Rajinikanth, N.S.M. Raja, S.C. Satapathy, "Robust color image multithresholding using between-class variance and cuckoo search algorithm," Advances in Intelligent Systems and Computing, vol. 433, pp. 379-386, 2016, https://doi.org/10.1007/978-81-322-2755-7_40.

[4] S.C. Satapathy, N.S.M. Raja, V. Rajinikanth, A.S. Ashour, N. Dey, "Multilevel image thresholding using Otsu and chaotic bat algorithm," Neural Computing and Applications, vol. 29, no. 12, pp. 1285-1307, 2018, https:// doi.org/10.1007/s00521-016-2645-5.

[5] A. Bahriye, "A study on particle swarm optimization and artificial bee colony algorithms for multilevel thresholding," Applied Soft Computing, vol. 13, no. 6, pp. 3066-3091, 2013, https://doi.org/10.1016/j. asoc.2012.03.072.

[6] P. Ghamisi, M.S. Couceiro, F.M.L. Martins, J.A. Benediktsson, "Multilevel image segmentation based on fractional-order Darwinian particle swarm optimization," IEEE Transactions on Geoscience and Remote sensing, vol. 52, no.5, pp. 2382-2394, 2014.

[7] S.L. Fernandes, V. Rajinikanth, S. Kadry, "A hybrid framework to evaluate breast abnormality using infrared thermal images," IEEE Consumer Electronics Magazine, vol. 8, no. 5, pp. 31-36, 2019, doi: 10.1109/ MCE.2019.2923926.

[8] M. Sezgin, B. Sankar, "Survey over Image Thresholding Techniques and Quantitative Performance Evaluation," Journal of Electronic Imaging, vol. 13, no. 1, pp. 146- 165, 2004.

[9] M. Tuba, "Multilevel image thresholding by nature-inspired algorithms: A short review," Computer Science fournal of Moldova, vol. 22, no. 3, pp. 318-338, 2014.

[10] V. Rajinikanth, S.C. Satapathy, S.L. Fernandes, S. Nachiappan, "Entropy based segmentation of tumor from brain MR images-a study with teaching learning based optimization," Pattern Recognition Letters, vol. 94, pp. 87-95, 2017. https://doi.org/10.1016/j.patrec.2017.05.028.

[11] N. Arunkumar, K. Ramkumar, V. Venkatraman, E. Abdulhay, S.L. Fernandes, S. Kadry, S. Segal, "Classification of focal and non focal EEG using entropies," Pattern Recognition Letters, vol. 94, pp. 112-117, 2017, https://doi.org/10.1016/j.patrec.2017.05.007.

[12] S.Z. Abbas, W.A. Khan, S. Kadry, M. Ijaz Khan, M. Waqas, M. Imran Khan, "Entropy optimized Darcy-Forchheimer nanofluid (Silicon dioxide, Molybdenum disulfide) subject to temperature dependent viscosity," Computer Methods and Programs in Biomedicine, vol. 190, 105363, 2020, https://doi.org/10.1016/j.cmpb.2020.105363

[13] S.Z. Abbas, M. Ijaz Khan, S. Kadry, W.A. Khan, M. Israr-Ur-Rehman, M. Waqas, "Fully developed entropy optimized second order velocity slip MHD nanofluid flow with activation energy," Computer Methods and Programs in Biomedicine, vol. 190, 105362, https://doi.org/10.1016/j. cmpb.2020.105362.

[14] S. Agrawal, R. Panda, S. Bhuyan, B.K. Panigrahi, "Tsallis entropy based optimal multilevel thresholding using cuckoo search algorithm," Swarm and Evolutionary Computation, vol. 11, pp. 16-30, 2013.

[15] N.S.M. Raja, V. Rajinikanth, K. Latha, "Otsu based optimal multilevel image thresholding using firefly algorithm," Modelling and Simulation in Engineering, vol. 2014, 794574, 2014, https://doi.org/10.1155/2014/794574.

[16] V. Rajinikanth and M.S. Couceiro, "Optimal multilevel image threshold selection using a novel objective function," Advances in Intelligent Systems and Computing, vol. 340, pp. 177-186, 2015, https://doi.org/10.1007/97881-322-2247-7_19.

[17] P. D. Sathya, R. Kalyani, V. P. Sakthivel, "Color image segmentation using Kapur, Otsu and Minimum Cross Entropy functions based on Exchange 
Market Algorithm," Expert Systems with Applications, vol. 172, 114636, 2021.

[18] T. R. Farshi and A.K. Ardabili, "A hybrid firefly and particle swarm optimization algorithm applied to multilevel image thresholding," Multimedia Systems, vol. 27, no. 1, pp. 125-142, 2021.

[19] J. Anitha, S.I.A. Pandian, S.A. Agnes, "An efficient multilevel color image thresholding based on modified whale optimization algorithm," Expert Systems with Applications, vol. 178, 115003, 2021.

[20] R. Kurban, A. Durmus, E. Karakose, "A comparison of novel metaheuristic algorithms on color aerial image multilevel thresholding," Engineering Applications of Artificial Intelligence, vol. 105, 104410, 2021.

[21] A.K. Bhandari, "A novel beta differential evolution algorithm-based fast multilevel thresholding for color image segmentation," Neural Computing and Applications, vol. 32, no. 9, pp. 4583-4613, 2020.

[22] Z. Xing, "An improved emperor penguin optimization based multilevel thresholding for color image segmentation," Knowledge-Based Systems, vol. 194, 105570, 2020.

[23] S. Meyyappan, S. Sathishbabu, N. Vinoth, M. Vijayakarthick, A.G. Ram, "Thresholding of Skin Melanoma Images based on Kapur's Entropy with Harmony Search Algorithm," European fournal of Molecular \& Clinical Medicine, vol. 7, no. 11, pp. 716-726, 2020.

[24] S. Borjigin and P.K. Sahoo, "Color image segmentation based on multilevel Tsallis-Havrda-Charvát entropy and 2D histogram using PSO algorithms," Pattern Recognition, vol. 92, pp. 107-118, 2019.

[25] S. Kadry and V. Rajinikanth, "Grey Scale Image Multi-Thresholding Using Moth-Flame Algorithm and Tsallis Entropy," furnal Ilmiah Teknik Elektro Komputer dan Informatika (FITEKI), vol. 6, no. 2, pp. 79-89, 2020.

[26] M. Abd Elaziz, N. Nabil, R. Moghdani, A.A. Ewees, E. Cuevas, S. Lu, "Multilevel thresholding image segmentation based on improved volleyball premier league algorithm using whale optimization algorithm," Multimedia Tools and Applications, vol. 80, no. 8, pp. 12435-12468, 2021.

[27] M. Abd Elaziz, A.A. Heidari, H. Fujita, H. Moayedi, "A competitive chainbased Harris Hawks Optimizer for global optimization and multi-level image thresholding problems," Applied Soft Computing, vol. 95, 106347, 2020.

[28] J.N. Kapur, P.K. Sahoo, A.K.C Wong, "A new method for gray-level picture thresholding using the entropy of the histogram," Comput Vision Graph Image Process, vol. 29, pp. 273-285, 1985.

[29] C. Tsallis, "Possible generalization of Boltzmann-Gibbs statistics," fournal of Statistical Physics, vol. 52, pp. 479-487, 1988.

[30] S. Mirjalili, "Moth-flame optimization algorithm: A novel nature-inspired heuristic paradigm," Knowledge-Based Systems, vol. 89, pp. 228-249, 2016, https://doi.org/10.1016/j.knosys.2015.07.006.

[31] S. J. Nanda, "Multi-objective moth flame optimization," In 2016 International conference on Advances in computing, communications and informatics (ICACCI), IEEE, 2016, pp. 2470-2476.

[32] M. Shehab, L. Abualigah, H. Al Hamad, H. Alabool, M. Alshinwan, A. M. Khasawneh, "Moth-flame optimization algorithm: variants and applications," Neural Computing and Applications, vol. 32, pp.9859-9884, 2020, https://doi.org/10.1007/s00521-019-04570-6.

[33] S.H.H. Mehne and S. Mirjalili, "Moth-Flame Optimization Algorithm Theory, Literature Review, and Application in Optimal Nonlinear Feedback Control Design," Nature-Inspired Optimizers, vol. 811, pp. 143166, 2020, https://doi.org/10.1007/978-3-030-12127-3_9.

[34] S. Grgic, M. Grgic, M. Mrak, "Reliability of objective picture quality measures," Journal of Electrical Engineering, vol. 55, no. 1-2, pp. 3-10, 2004.

[35] Z. Wang, A.C. Bovik, H.R. Sheikh, E.P. Simoncelli, "Image Quality Assessment: From Error Visibility to Structural Similarity," IEEE Transactions on Image Processing, vol. 13, no. 4, pp. 600- 612.

[36] A. Hemeida, R. Mansour, M.E. Hussein, "Multilevel Thresholding for Image Segmentation Using an Improved Electromagnetism Optimization Algorithm," International fournal of Interactive Multimedia and Artificial Intelligence, vol. 5, no. 4, pp. 102-112, http://doi.org/10.9781/ ijimai.2018.09.001

[37] S. Kadry, V. Rajinikanth, J. Koo, B.G. Kang, "Image multi-levelthresholding with Mayfly optimization," International fournal of Electrical \& Computer Engineering, vol. 11, no. 6, pp. 5420-5429, 2021.

[38] V. Rajinikanth, S.M. Aslam, S. Kadry, O. Thinnukool, "Semi/FullyAutomated Segmentation of Gastric-Polyp Using Aquila-OptimizationAlgorithm Enhanced Images," Cmc-Computers Materials \& Continua, vol. 70, no. 2, pp. 4087-4105, 2022.

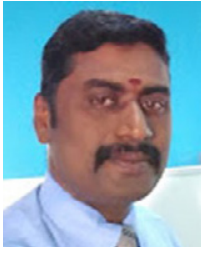

\section{Rajinikanth}

V. Rajinikanth is a Professor in Department of Electronics and Instrumentation Engineering at St. Joseph's College of Engineering, Chennai 600119, Tamilnadu, India. He has published more than 125 papers and authored/edited 7 books in the field of medical data assessment. His main research interests include Heuristic algorithm based optimization, Image thresholding, Machine learning and

Deep learning.

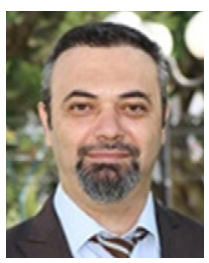

\section{Seifedine Kadry}

Seifedine Kadry (Senior Member, IEEE) received the bachelor's degree from Lebanese University, in 1999, the dual M.S. degree from Reims University, France, and EPFL, Lausanne, in 2002, the Ph.D. degree from Blaise Pascal University, France, in 2007, and the H.D.R. degree from Rouen University, in 2017. He is an IET Fellow, IETE Fellow. His research interests include data science, education using technology, system prognostics, stochastic systems, and applied mathematics. Currently, he is a full professor of data science at Noroff University College, Kristiansand, Norway.

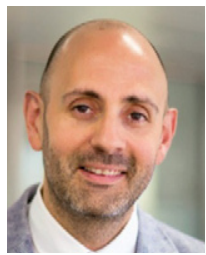

\section{Rubén González Crespo}

Dr. Rubén González Crespo has a PhD in Computer Science Engineering. Currently he is Vice Chancellor of Academic Affairs and Faculty from UNIR and Global Director of Engineering Schools from PROEDUCA Group. He is advisory board member for the Ministry of Education at Colombia and evaluator from the National Agency for Quality Evaluation and Accreditation of Spain (ANECA). $\mathrm{He}$ is member from different committees at ISO Organization. Finally he has published more than 200 paper in indexed journals and congresses.

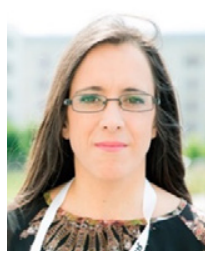

\section{Elena Verdú}

Elena Verdú received the master's and Ph.D. degrees in Telecommunications Engineering from the University of Valladolid, Spain, in 1999 and 2010, respectively. She is currently an Associate Professor with the Universidad Internacional de La Rioja (UNIR), where she is also a member of the Research Group "Data Driven Science." For more than 15 years, she has worked on research projects at both national and European levels. Her research has focused on e-learning technologies, intelligent tutoring systems, competitive learning systems, data mining, machine learning, natural language processing, image and speech processing, and expert systems. 